\title{
HMFG-2 as a prognostic indicator in superficial bladder cancer
}

\author{
I G CONN, $\ddagger \S$ J CROCKER, $†$ L A EMTAGE, $\ddagger$ D M A WALLACE $\S$
}

From the *Surgical Immunology Unit, $\ddagger$ Cancer Research Campaign Clinical Trials Unit, and $\S$ Department of Urology, Queen Elizabeth Hospital, Birmingham, and the †Department of Histopathology, East Birmingham Hospital, Birmingham

SUMMARY A series of transitional cell carcinomas and mucosal biopsy specimens of bladder were stained immunohistochemically with the monoclonal antibody HMFG-2. Staining characteristics ranged from luminal staining in well differentiated, superficial lesions to staining of all cells in invasive carcinomas. Invasive tumour nests also stained strongly with the antibody. There was good correlation between the staining pattern and histological assessment of both tumours and mucosal biopsy specimens. Correlation with clinical course showed that it was also a good predictor of subsequent disease progression of superficial tumours.

It is suggested that HMFG-2 staining is a sensitive indictor of malignant transformation and that it may have a role as an adjunct to conventional histological examination in the assessment of urothelial carcinomas.

Superficial bladder cancers vary widely in their propensity to recur or invade deeply. Once diagnosed as having superficial bladder cancer, a patient has about a $70 \%$ chance of developing recurrences, either at the site of the primary lesion or at another site in the urinary tract, and about a $10-20 \%$ chance of progression to muscle invasive disease. ${ }^{12}$ Several characteristics of the initial tumour have been correlated with clinical course and eventual outcome. Stromal invasion, poor histological grade, large and multiple tumours have all been associated with an increased risk of recurrence and progression..$^{3-5}$ Although these clinical and pathological variables can give some indication of prognosis and are of value in the planning of follow up requirements, more accurate methods of identifying the "high risk" bladder are required if prophylaxis, such as intravesical chemotherapy or early radical surgery, is to be considered.

An antiserum raised against epithelial membrane antigen (EMA), a large glycoprotein component of human milk fat globule (HMFG) membrane, has previously been shown to exhibit a changed staining pattern in urothelial malignancy. ${ }^{6}$ The monoclonal antibody HMFG-2 is directed against a much smaller carbohydrate residue on the HMFG membrane which, although present as a normal feature of the luminal surface, also seems to be a result of changed cell surface glycosylation in malignant transformation. ${ }^{7}$
This study was performed to assess the value of immunohistochemical staining with HMFG-2 as an aid to standard histological techniques in the assessment of superficial bladder tumours and urothelial mucosal biopsy specimens.

\section{Material and methods}

RETROSPECTIVE STUDY

Eighty four transitional cell carcinomas of the bladder (69 superficial, 15 invasive), which were initially resected in the Queen Elizabeth Hospital, Birmingham between 1980 and 1982, were studied. All the blocks had been fixed in $10 \%$ formol saline, wax embedded, and were archived in the pathology department. Sections of $3 \mu \mathrm{m}$ thickness were cut from each tumour block and processed for immunohistochemical staining as described below. Hospital case records and cancer registry follow up notes were scrutinised to obtain five year follow up information on recurrence and progression for all the patients. Progression was defined as an increase in $\mathrm{pT}$ category from $\mathrm{pTa}$ or $\mathrm{pT} 1$ to muscle invasive disease (pT2+), or the development of metastatic disease.

\section{PROSPECTIVE STUDY}

Twenty six patients attending for cystoscopy under general anaesthetic either as follow up for superficial bladder tumours before transurethral resection of the prostate were studied. Either a random mucosal biopsy or, if a tumour was present, near-far biopsy 
specimens from macroscopically normal mucosa ${ }^{8}$ were taken using cold-cup biopsy forceps, fixed in $10 \%$ formaldehyde in phosphate buffered saline and embedded in paraffin wax. Sections of $3 \mu \mathrm{m}$ thickness were then cut from each block for immunohistochemical analysis.

\section{IMMUNOCHEMISTRY}

The HMFG-2 mouse monoclonal antibody was obtained from Oxoid-Unipath Ltd, Viking Industrial Estate, Bedford, United Kingdom. After initial dewaxing and rehydration the sections were stained by the indirect immunoperoxidase technique.' Briefly, they were incubated with the HMFG-2 antibody, diluted 1:100 in phosphate buffered saline with $1 \%$ bovine serum albumin and then with rabbit antimouse peroxidase conjugate for $\mathbf{3 0}$ minutes each. Peroxidase activity was visualised using diaminobenzidine.

CLASSIFICATION AND SCORING OF HISTOLOGICAL SECTIONS

Sections stained with haematoxylin and eosin were examined and each tumour classified according to stromal or muscular invasion ${ }^{10}$ and degree of histological differentiation." The sections stained for HMFG-2 were given a score of 1-3, depending on the depth of penetration of stain from the luminal surface to the basal cells of the epithelium: 1 = staining of luminal cells alone (fig 1);2 = staining of luminal and

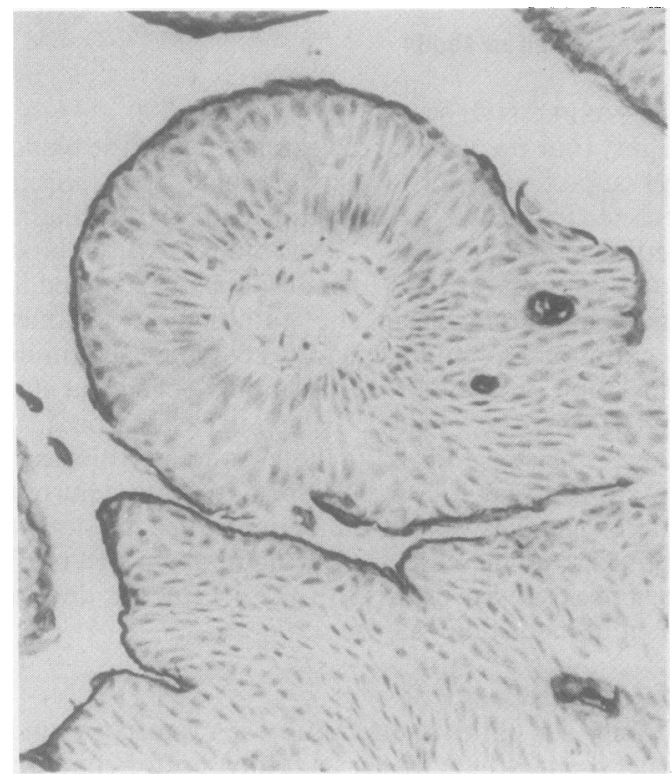

Fig 1 Well differentiated (GI) pTa transitional cell carcinoma of bladder. Staining of luminal cells alone (HMFG-2). intermediate cells (fig 2); 3 = staining of all epithelial cells (fig 3).

Statistical analysis was performed using programs

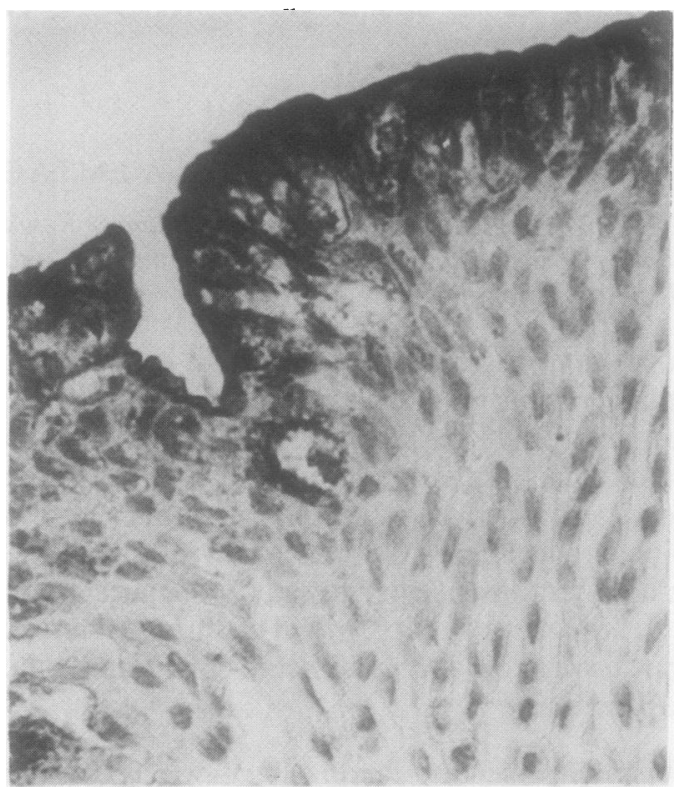

Fig 2 Moderately differentiated (G2) pTa transitional cell carcinoma of bladder. Staining of superficial and intermediate cells (HMFG-2).

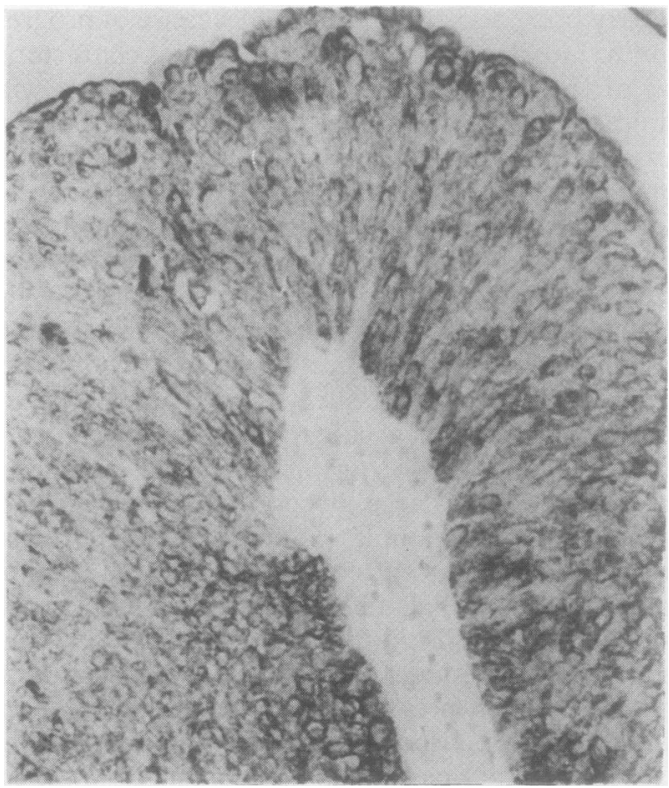

Fig 3 Moderately differentiated (G2) pTa transitional cell carcinoma of bladder. Staining of all epithelial cells (HMFG-2). 
from the BMDP (Biomedical Programs, Los Angeles, California) statistical package on the Systime VAX 11/ 730 minicomputer in the West Midlands Cancer Research Campaign Clinical Trials Unit. Actuarial survival curves for time to progression were drawn using the method of Kaplan and Meier ${ }^{12}$ and the difference between groups calculated using the logrank test. ${ }^{13}$

\section{Results}

\section{RETROSPECTIVE STUDY}

Of the 84 tumours, sections from eight were either too small or fragmented to permit adequate assessment of grade, stromal, or muscular invasion. The study was therefore performed on the remaining 76 tumours. All of the sections stained positively for HMFG-2. Staining characteristics ranged from luminal staining alone (score $=1$ ) in 16, staining of superficial and intermediate cells (score $=2$ ) in 38 , to staining of all the cells (score $=3$ ) in 22 . There was a significant association between pT category and HMFG-2 score (table 1). Of $36 \mathrm{pTa}$ tumours, $13(36 \%)$ had a score of 1 and only seven $(19 \%)$ had a score of 3 . Where there was histological evidence of stromal invasion (pT1) only two of $27(7 \%)$ had a score of 1 and the remainder had some degree of penetration of stain towards the basement membrane. Of 13 muscle invasive tumours, only one had a score of 1 ; eight $(62 \%)$ had a score of 3 . Table 2 shows that there was also a significant association between HMFG-2 score and histological grade, with poorly differentiated lesions having deeper penetration of stain towards the basement membrane. Strong staining was also seen in nests of invasive tumour cells in the submucosa (fig 4). In three tumours, which were regarded as pTa by conventional

Table 1 HMFG-2 staining and $p T$ category

\begin{tabular}{llll}
\hline \multicolumn{4}{c}{$H M F G-2$ score } \\
\cline { 2 - 4 } & 1 & 2 & 3 \\
\hline pTa & 13 & 16 & 7 \\
pT1 & 2 & 18 & 7 \\
pT2 & 1 & 4 & 8 \\
\hline
\end{tabular}

$\left(x^{2}(4 d f)=16 \cdot 0, p=0.003\right)$.

Table 2 HMFG-2 staining and histological grade

\begin{tabular}{llrr}
\hline & \multicolumn{4}{l}{ HMFG-2 score } \\
\cline { 2 - 4 } & 1 & 2 & 3 \\
\hline G1 & 9 & 9 & 6 \\
G2 & 7 & 16 & 5 \\
G3 & 0 & 13 & 11 \\
\hline
\end{tabular}

$\left(\chi^{2}(4 d f)=13 \cdot 2, p=0.01\right)$. histological criteria, this showed probable stromal invasion.

The association between HMFG-2 staining and clinical course was further examined for the 63 superficial tumours in the study. Although there was poor correlation between HMFG-2 staining and recurrence $\left(\chi^{2}(2 \mathrm{df})=4.7 \mathrm{p}=0.09\right)$, there was a significant correlation between staining pattern and subsequent progression to muscle invasive or

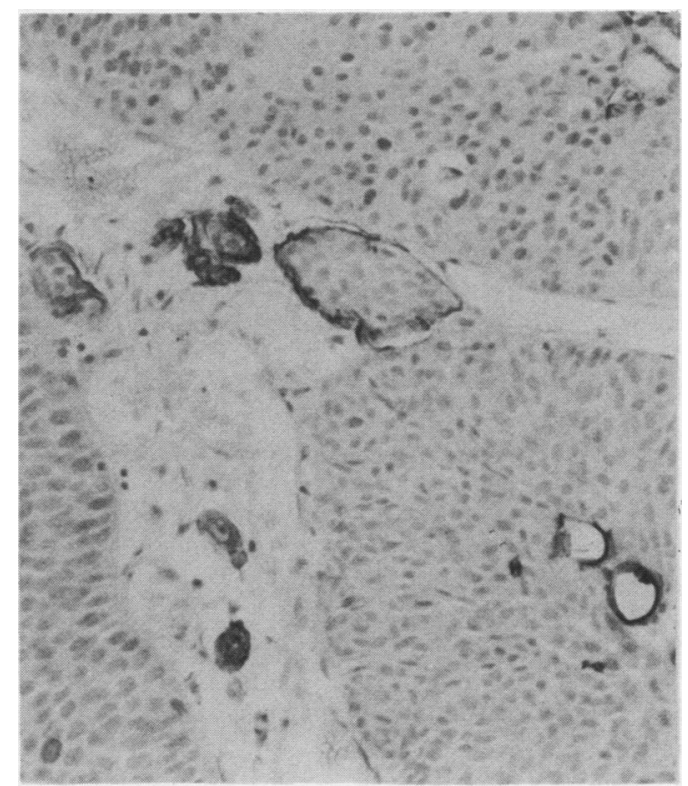

Fig 4 Moderately differentiated (G2) pTl transitional cell carcinoma of bladder. Strong staining of invasive nests (HMFG-2).

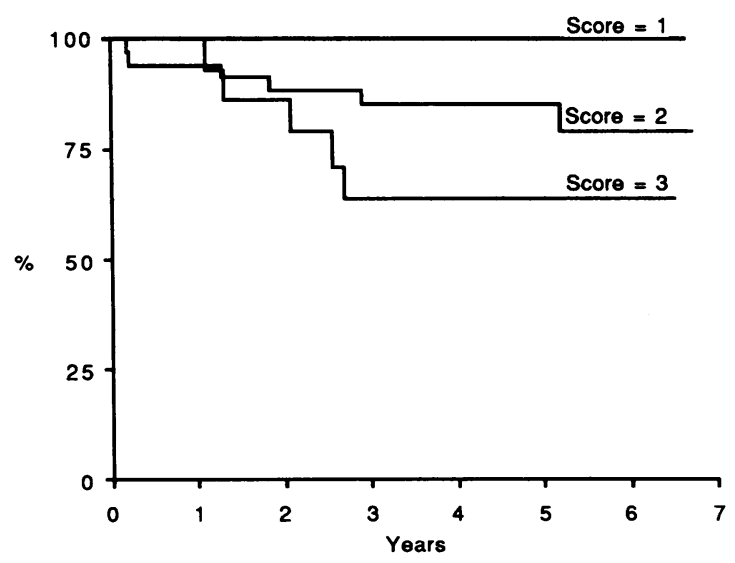

Fig 5 Effect of HMFG-2 staining on disease progression: score $=1 / 0 / 15(0 \%)$ progressed; score $=2 / 6 / 34(18 \%)$ progressed; score $=3 / 5 / 14(36 \%)$ progressed; $\chi^{2}(2 d f)=6.1 p=0.047$. 
Table 3 HMFG-2 staining of mucosal biopsy specimens

\begin{tabular}{lccc}
\hline & \multicolumn{4}{l}{ HMFG-2 score } \\
\cline { 2 - 4 } & 1 & 2 & 3 \\
\hline Normal & 9 & 8 & 0 \\
Hyperplasia & 0 & 1 & 2 \\
Mild dysplasia & 6 & 3 & 1 \\
Severe dysplasia & 1 & 1 & 0 \\
Carcinoma in situ & 0 & 0 & 3 \\
& 16 & 13 & 6 \\
\hline
\end{tabular}

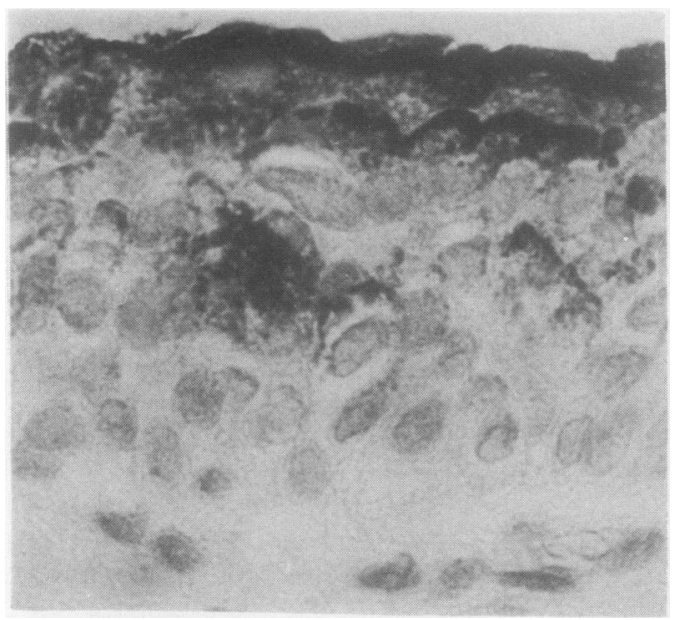

Fig 6 Mild dysplasia. Staining of luminal and intermediate cells (HMFG-2).

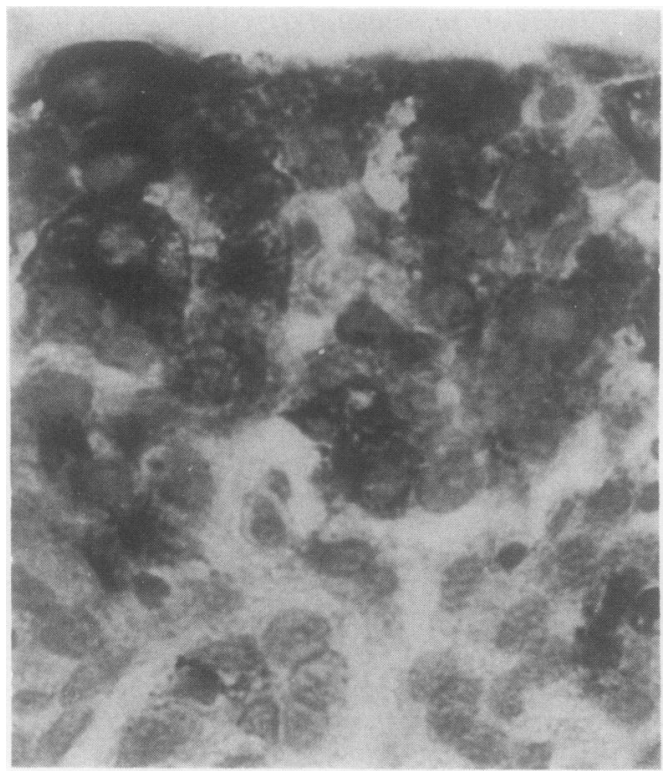

Fig 7 Carcinoma in situ. Staining of all epithelial cells (HMFG-2). metastatic disease (fig 5). Of 15 tumours with staining of the luminal cells alone (score $=1$ ), none progressed; six of $34(18 \%)$ with stain penetrating towards the basement membrane (score $=2$ ) and five of $14(36 \%)$ with staining of all the epithelial cells (score $=3$ ) progressed.

\section{PROSPECTIVE STUDY}

Of 43 mucosal biopsy specimens examined, eight were unsuitable for analysis because they were too small or traumatised. The study was therefore performed on the remaining 35 specimens (table 3 ). Seventeen were histologically normal and of these, nine had luminal staining alone; eight had some penetration of stain through the epithelium (fig 6). None had staining of all the epithelial cells. The remaining 18 specimens showed some degree of premalignant change and, of these, 11 had a score of 2 or 3 . All three biopsy specimens of carcinoma in situ had staining throughout the epithelial layer (fig 7).

\section{Discussion}

The fat globules released into milk during lactation are surrounded by a membrane which is representative of the terminally differentiated mammary epithelial cell. These membranes are easily obtainable from the cream fraction of milk and have been widely used to produce both polyclonal antisera and monoclonal antibodies which are specific for epithelial cells. ${ }^{14}$ is The most immunogenic component of HMFG is a large mucin-like glycoprotein with molecular weight of around $\mathbf{4 4 0} 000$. Heyderman et al first described EMA, identified by an antiserum raised against HMFG membrane, which was localised to membranes with secretory function. ${ }^{16}$ They showed positive staining with this antibody in bladder, prostatic, and colorectal neoplasms. ${ }^{17}$ Further work showed that EMA was confined to the luminal surface of normal epithelial tissues but, in inflammatory and neoplastic conditions, staining increased and penetrated more deeply into the epithelium. Micrometastases also stained positively with the antibody. ${ }^{18}$ The potential usefulness of EMA staining in urothelial carcinoma was suggested by Pocock et al, who correlated its staining pattern with pT category, grade, and subsequent clinical course. ${ }^{6}$ They showed luminal staining only in normal epithelium but penetration down towards the submucosa in many cases of neoplasia.

HMFG-1 and HMFG-2 are monoclonal antibodies raised against components of the HMFG membrane. These have been used to differentiate carcinomas of epithelial origin from mesotheliomas and reactive mesothelium. ${ }^{19}$ The HMFG-1 determinant is a large glycoprotein molecule with complex carbohydrate side chains whereas HMFG-2 is active against smaller carbohydrate molecules. Its expression is thought to be associated with incomplete or changed glycosylation in malignant change. 
Our results show a strong correlation between HMFG-2 staining and both extent of invasion and histological grade. A high HMFG-2 score was also associated with a significantly higher risk of progression to muscle invasive or metastatic disease $(\mathrm{p}=$ 0.047 ). Greater penetration of the stain towards the basement membrane therefore seems to be associated with an increasing degree of malignant transformation of the epithelial cells. The mucosal biopsy specimens also expressed a similar relation, with most normal biopsy specimens having luminal staining alone; dysplastic or neoplastic epithelium had deep penetration of staining.

In developing malignancy, therefore, intermediate and then basal cells progressively express this antigen which is a normal feature of the luminal membrane of urothelial cells. One explanation for this appearance is that the changed cell surface glycosylation associated with malignant transformation is similar to the change which develops in the terminally differentiated superficial cell. Another possible explanation, however, is that the HMFG-2 antibody is directed against a secretory mucin on the luminal cell surface. Intercellular adhesion has been shown to decrease with malignant change ${ }^{20}$ and this would allow penetration of mucin in between the superficial and intermediate cells to give the characteristic appearance described above. In the poorly differentiated tumour both loss of polarity and decreased cell to cell adhesion may contribute to the diffuse HMFG-2 staining throughout the epithelial layer. The fact that the antibody is raised against a secretory product - the milk fat globule - strengthens this argument.

HMFG-2 is immunoreactive in routinely fixed, paraffin wax embedded material without the need for protease pretreatment. In this study HMFG-2 staining identified a group of patients who were at higher risk of disease progression and, as such, may have benefited from an early aggressive treatment policy. A low risk group was also identified in whom follow up requirements could possibly have been reduced. A range of staining was also seen in mucosal biopsy specimens, possibly reflecting early malignant change.

We conclude that HMFG-2 staining is a sensitive indicator of malignant transformation in urothelial carcinoma. A larger, prospective study would, however, be required to assess the implications of HMFG-2 staining on patient management.

Grateful thanks are due to Dr F Macdonald, Mrs HJ Stokes, and Mrs B Russell of the surgical immunology unit for their helpful advice and willing assistance. We are also most grateful to Professor EL Jones for allowing us to study archived material from the pathology department, University of Birmingham Medical School.

Mr IG Conn is supported by the Cancer Research Campaign and the project was supported by a grant from the Central Birmingham Endowment Trust Fund.
References

1 Boyd PRJ, Burnand KG. Site of bladder tumour recurrence. Lancet 1974;ii: 1290-2.

2 Lutzeyer W, Rubben H, Dahm H. Prognostic parameters in superficial bladder cancer: An analysis of 315 cases. J Urol 1982;127:250-2.

3 Pocock RD, Ponder BAJ, O'Sullivan JP, Ibrahim SK, Easton DF, Shearer RJ. Prognostic factors in non-infiltrating carcinoma of the bladder: a preliminary report. Br J Urol 1982;54:711-5.

4 Heney NM, Nocks BN, Daly JJ, et al. Ta and Tl bladder cancer: location, recurrence and progression. Br J Urol 1982;54:152-7.

5 Dalesio O, Shulman CC, Sylvester R, et al. Prognostic factors in superficial bladder tumors. A study of the European Organisation for Research on Treatment of Cancer: Genitourinary Tract Cooperative Group. J Urol 1983;129:730-3.

6 Pocock RD, Ibrahim SK, Sloane JP, Ponder BAJ, Shearer RJ. Potential value of antisera to epithelial membrane antigen in detecting early invasion in transitional cell carcinoma. Br J Urol 1983;55:670-5.

7 Burchell J, Durbin H, Taylor-Papadimitriou J. Complexity of expression of antigenic determinants, recognised by monoclonal antibodies HMFG-1 and HMFG-2 in normal and malignant human mammary epithelial cells. J Immunol 1983;131:508-13.

8 Hargreave TB. Carcinoma in situ. In: Zingg EJ, Wallace DMA, eds. Bladder cancer. Berlin. Springer-Verlag, 1985;141-60.

9 Heyderman E. Immunoperoxidase techniques in pathology: applications, methods and controls. J Clin Pathol 1979;32: 971-8.

10 UICC. Hermanek P, Sobin LH, eds. TNM classification of malignant tumours. 4th ed. Berlin; Springer-Verlag, 1987.

11 Mostofi FK, Sobin LH, Torloni H. Histological typing of urinary bladder tumours. International histological classification of tumours. No 10. Geneva: World Health Organisation, 1973.

12 Kaplan EL, Meier P. Nonparametric estimation from incomplete observations. J Am Statis Assoc 1958;53:457-81.

13 Peto R, Pike MC, Armitage P, et al. Design and analysis of randomised clinical trials requiring prolonged observation of each patient. II. Analysis and examples. Br J Cancer 1977;35: 1-3.

14 Taylor-Papadimitriou J, Peterson JA, Arklie J, Burchell J, Ceriani RL, Bodmer WF. Monoclonal antibodies to epithelium specific components of the human milk fat globule membrane: production and reaction with cells in culture. Int J Cancer 1981;28:1721.

15 Heyderman E, Strudley I, Powell G, Richardson TC, Cordell JL, Mason DY. A new monoclonal antibody to epithelial membrane antigen (EMA)-E29. A comparison of its immunocytochemical reactivity with polyclonal anti-EMA antibodies and with another monoclonal antibody, HMFG-2. Br J Cancer 1985;52:355-61.

16 Heyderman E, Steele K, Ormerod MG. A new antigen on the epithelial membrane: its immunoperoxidase localisation in normal and neoplastic tissue. J Clin Pathol 1979;32:35-9.

17 Heyderman E, Brown BME, Richardson TC. Epithelial markers in prostatic, bladder, and colorectal cancer: An immmunoperoxidase study of epithelial membrane antigen carcinoembryonic antigen, and prostatic acid phosphatase. $J$ Clin Pathol 1984;37:1363-9.

18 Sloane JP, Ormerod MG. Distribution of epithelial membrane antigen in normal and neoplastic tissues and its value in diagnostic tumour pathology. Cancer 1981;47:1786-95.

19 Marshall RJ, Herbert A, Braye SG, Jones DB. Use of antibodies to carcinoembryonic antigen and human milk fat globule to distinguish carcinoma, mesothelioma, and reactive mesothelium. J Clin Pathol 1984;37:1215-21.

20 Alroy J, Pauli BU, Weinstein RS. Correlation between numbers of desmosomes and the aggressiveness of transitional cell carcinoma in human urinary bladder. Cancer 1981;47:104-12.

Requests for reprints to: Mr IG Conn, Department of Surgery/Urology, Western General Hospital, Edinburgh EH4 2XU, Scotland. 